\title{
TEM of Paraffin-Embedded H\&E-Stained Sections for Viral Diagnosis (An Unusual Papovavirus Case)
}

Januario C. Estrada, M. Angelica Selim, and Sara E. Miller

Department of Pathology, Duke University Medical Center, Durham, NC 27710

Viral infections can be focal and therefore difficult to find by electron microscopy. In addition to sampling limitations, sometimes the only specimen available for examination is tissue that has already been prepared for light microscopy (LM). We describe the diagnosis of a papovavirus infection in skin by embedding hematoxylin and eosin (H\&E)-stained sections for ultrathin sectioning and transmission electron microscopy (TEM).

Several methods have been published for enhancing the possibility of locating focal lesions and for processing thin specimens. Localization of focal infections in wet tissue can be achieved by confocal microscopy; excised areas are then processed for TEM [1]. Monolayers of cell cultures have been processed in situ for thin sections [2]. Wax can be removed from paraffin-embedded blocks for further processing of tissue for TEM and other studies [3, 4], but a focal infection might be missed. The processing of unstained frozen or wax sections (hence, unidentified lesions) for TEM has been described, but not for viral diagnosis [5]. The technique discussed here shows that some viruses can still be identified by TEM in tissue that has been processed through the harsh treatment for LM examination.

Immunosuppressed patients may contract unusual agents or infections in unusual places. Previously, we reported a case of a skin infection by a $40-\mathrm{nm}$ papovavirus [6]. In the case presented here, a viral diagnosis was suspected, but an accurate identification would have been missed without TEM. Molluscum contagiosum was considered based on location of the infection and LM of a lesion, but the primarily nuclear inclusions prompted further investigation. Molluscum, a poxvirus seen in skin of immunosuppressed patients, is a virus that replicates in the cytoplasm. In this case, inclusions were observed in the nucleus.

Since the only available tissue was that which had already been cut in 6-micron sections and processed by H\&E staining (Fig. 1), we removed the coverslip with xylene, scored the slide with a diamond-tipped pencil, and broke it to preserve some sections for LM. We then embedded the remaining sections on one end of the slide for TEM.

The deparaffinized sections were treated in situ on the slide by rehydration with descending alcohols, rinsing in cacodylate buffer, fixation in $4 \%$ glutaraldehyde, post-fixation in $1 \%$ osmium, re-dehydration with a graded series of ethanols and propylene oxide, and infiltration with Poly/Bed 812. A thin layer $(\sim 1 \mathrm{~mm})$ of resin was left on the slide, and it was baked overnight at $60^{\circ} \mathrm{C}$. Following polymerization, the glass slide and layer of resin were warmed in a beaker of water that had been heated to boiling and removed from the burner. Separation of the two was initiated with a razor blade and finished by simply pulling up the pliable resin layer while warm. It was laid flat, section side up, to cool. An area containing the tissue was cut out with a razor blade and glued onto a blank polymerized epoxy block for ultrathin sectioning. 
One might assume that the paraffin embedment, staining, and drying for LM and xylene treatment of the tissue to remove the wax and coverslip would render viral structure unrecognizable. However, in the case of 40-nm naked icosahedral viruses, they remain intact enough for identification (Fig. 2). Other viruses, such as smaller $(25-30 \mathrm{~nm})$ icosahedral viruses and enveloped viruses with helical nucleocapsids or morphologically indistinct nucleocapsids may not remain as easily recognizable.

We conclude that this method is useful for identifying many viruses by EM, even after previous harsh treatment of paraffin embedding, deparaffinization, LM staining, and air-drying.

\section{References}

[1] D. N. Howell, S. E. Miller. Meth. Enzymol. 307 (1999) 573.

[2] S. E. Miller. In B. R. Jones (ed.) Electron Microscopy, Library Research Associates, Inc., Monroe, NY (1985) p. 293.

[3] M. A. van den Bergh Weerman, K.P. Dingemans. Ultrastruct. Pathol. 7 (1984) 55.

[4] A. B. Maunsbach, B. A. Afzelius. Biomedical Electron Microscopy, Academic Press, New York (1999) p. 28.

[5[ K. G. Kupke et al. J. Electron Microsc. Tech. 1 (1984) 299.

[6] C. L. Haycox et al. J Investig. Dermatol. Symp. Proc. 4 (1999) 268.

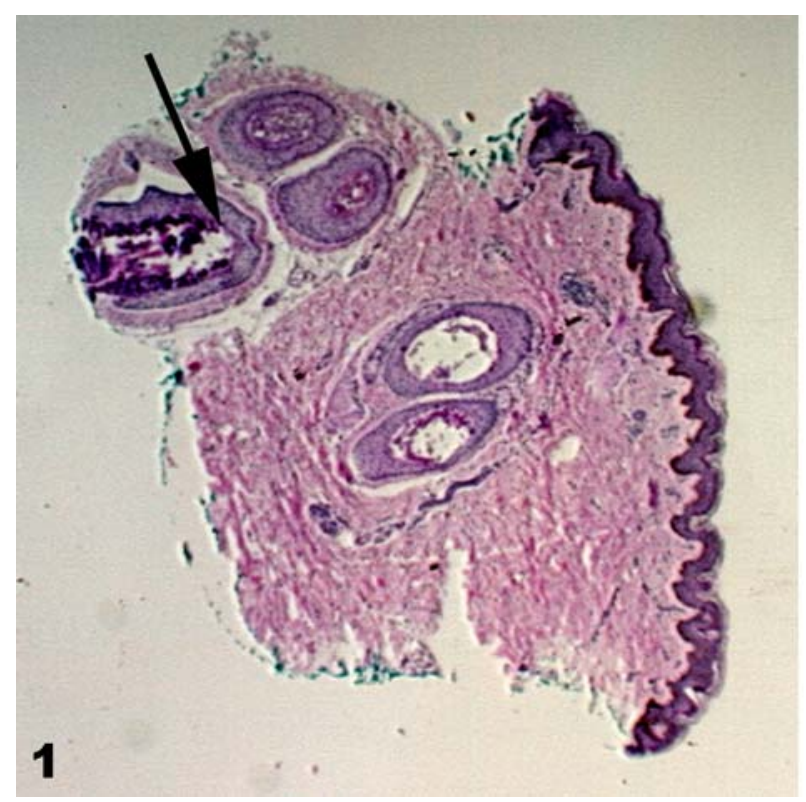

Figure 1. Light micrograph of H\&E-stained skin shows epithelium (right) and hair follicles. Arrow indicates area from which electron micrographs were taken.

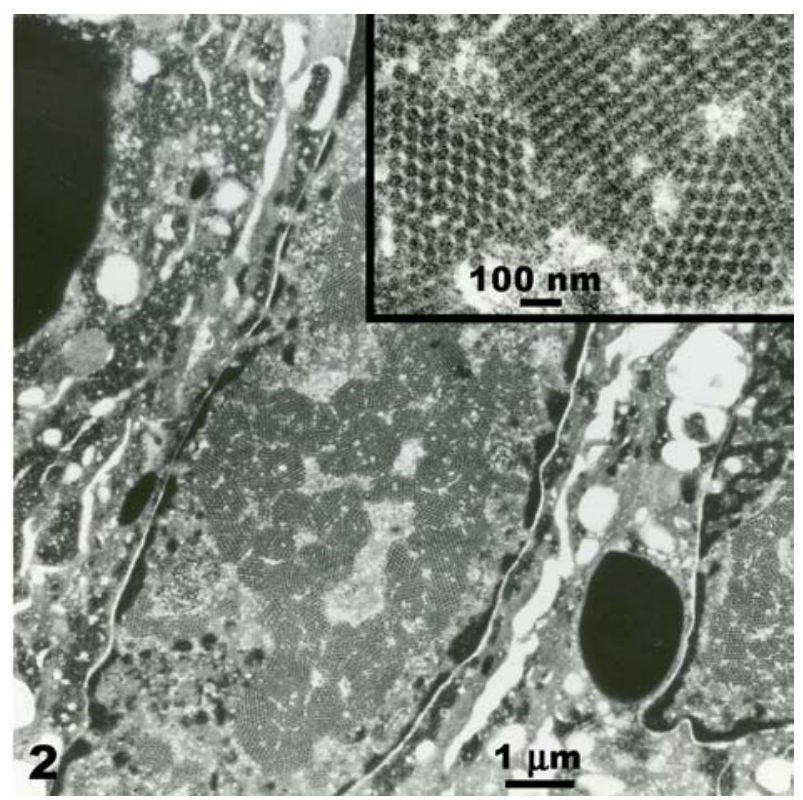

Figure 2. Low magnification electron micrograph shows nuclear inclusions of viruses. Inset shows high magnification of naked icosahedral viruses (papovaviruses). 